\title{
miR-140-5p alleviates mouse liver ischemia/reperfusion injury by targeting CAPN1
}

\author{
QIWEN YU ${ }^{1 *}$, SANYANG CHEN ${ }^{2 *}$, HONGWEI TANG $^{1}$, HAN YANG $^{1}$, JIAKAI ZHANG ${ }^{1}$, \\ XIAOYI SHI ${ }^{1}$, JIE LI ${ }^{1}$, WENZHI GUO ${ }^{1}$ and SHUIJUN ZHANG ${ }^{1}$ \\ Departments of ${ }^{1}$ Hepatobiliary and Pancreatic Surgery and ${ }^{2}$ Emergency, \\ The First Affiliated Hospital of Zhengzhou University, Zhengzhou, Henan 450052, P.R. China
}

Received February 9, 2021; Accepted July 5, 2021

DOI: $10.3892 / \mathrm{mmr} .2021 .12314$

\begin{abstract}
Ischemia/reperfusion (I/R)-induced liver injury remains a primary concern in liver transplantation and hepatectomy. Previous studies have indicated that microRNAs (miRs) are involved in multiple pathophysiological processes, including liver I/R. miR-140-5p reportedly inhibits inflammatory responses and apoptosis in several diseases; however, the role of miR-140-5p in liver I/R remains unknown. The present study aimed to investigate the potential role and mechanism of miR-140-5p on liver I/R injury. Mouse liver I/R and mouse AML12 cell hypoxia/reoxygenation (H/R) models were established. miR-140-5p mimics, inhibitor or agonists were used to overexpress or inhibit miR-140-5p in vitro and in vivo. Reverse transcription-quantitative polymerase chain reaction was used to detect miR-140-5p expression. Liver and cell injury were evaluated using several biochemical assays. The association between miR-140-5p and calpain-1 (CAPN1) was confirmed using a dual-luciferase reporter assay. The results revealed that miR-140-5p expression was decreased in the mouse model of liver I/R injury and AML12 cells subjected to H/R, while overexpressed miR-140-5p reduced liver injury in vivo and
\end{abstract}

Correspondence to: Dr Shuijun Zhang, Department of Hepatobiliary and Pancreatic Surgery, The First Affiliated Hospital of Zhengzhou University, 1 Jianshe East Road, Zhengzhou, Henan 450052, P.R. China E-mail: zhangshuijun@zzu.edu.cn

*Contributed equally

Abbreviations: I/R, ischemia/reperfusion; H/R, hypoxia reoxygenation; CAPN1, calpain 1; RT-qPCR, reverse transcription quantitative-polymerase chain reaction; 3'-UTRs, 3'-untranslated regions; NC, negative control; CCK-8, Cell counting Kit-8; LDH, lactate dehydrogenase; ALT, alanine aminotransferase; AST, aspartate aminotransferase; H\&E, hematoxylin and eosin; TUNEL, terminal deoxynucleotidyl transferase dUTP nick end labeling; GAPDH, glyceraldehyde 3-phosphate dehydrogenase; BCA, bicinchoninic acid; PVDF, polyvinylidene difluoride

Key words: liver ischemia/reperfusion injury, CAPN1, miR-140-5p, inflammation, apoptosis cell injury in vitro. In addition, CAPN1 was determined to be a target of miR-140-5p; overexpressed CAPN1 abrogated the effect of miR-140-5p on H/R-induced cell injury. The present study indicated that miR-140-5p protected against liver I/R by targeting CAPN1, which may provide a novel therapeutic target for liver I/R injury.

\section{Introduction}

Liver ischemia/reperfusion (I/R) injury is a common physiological and pathological process in liver surgery and liver transplantation. Clinically, liver I/R is often manifested as damage to liver function, increased incidence of postoperative complications, and mortality (1). Activation of Kupffer cells, oxidative stress, apoptosis, and activation of inflammatory signaling pathways are involved in the process of liver I/R injury (2). At present, commonly used clinical treatments include ischemic preconditioning, pharmacological preconditioning, and ischemic postconditioning, among others (3), but the effect is limited. Therefore, studies on the mechanism of liver I/R injury and its preventive measures are of great clinical significance for reducing the complications of liver surgery, improving the success rate of liver transplantation, and promoting the recovery of postoperative liver function.

MicroRNAs (miRNAs or miRs) are a class of highly conserved, endogenous, small non-coding RNAs that play important regulatory roles in a variety of biological processes and usually inhibit the expression of target genes by binding to the 3'-untranslated regions (3'-UTRs) of target mRNAs (4). In liver I/R injury, miRNAs, such as miR-125b, miR-128-3p, miR-142-3p, miR-450b-5p, and miR-27a, participate in the regulation of liver $\mathrm{I} / \mathrm{R}$ injury through inflammatory responses, oxidative stress, apoptosis, autophagy, and energy metabolism (5-9).

miR-140-5p, also known as miR-140, was first identified in zebrafish in 2005 (10). In 2006, Tuddenham et al reported that miR-140-5p was a cartilage-specific miRNA in mouse cells (11). In 2009, Miyaki et al revealed that miR-140-5p was highly expressed in differentiated human articular chondrocytes (12), while later studies reported that miR-140-5p was also expressed in other human tissues. miR-140-5p has been revealed to be expressed at low levels in various tumor tissues and play an antitumor role (13-18). miR-140-5p has been revealed to inhibit inflammatory response (19-21) and 
oxidative stress in non-tumor diseases $(22,23)$, and also be involved in the regulation of brain I/R injury (24).

The calpain family members are calcium-dependent proteases that regulate numerous cellular functions by truncating bound proteins, leading to changes in their properties and functions (25). Overactivation of calpain has been revealed to be involved in numerous diseases. The activity and expression of calpain-1 (CAPN1) has been revealed to increase after ischemia in the heart, liver, brain and other organs, while CAPN1 inhibition has been reported to alleviate organ I/R injury (26-28).

In the present study, the expression and effect of miR-140-5p in mouse liver tissues following I/R injury and in H/R-induced AML12 cells were investigated. It was predicted that CAPN1 may be a target of miR-140-5p, and thus the regulatory effect of miR-140-5p on CAPN1 was demonstrated. Our novel insights into miR-140-5p may provide a new therapeutic option for liver I/R injury.

\section{Materials and methods}

Animals and liver I/R model. Male C57BL/6 mice $(\mathrm{n}=54)$ weighing 20-24 g (6-8 weeks old) were purchased from Huaxin Laboratory Animal Co., Ltd. All the mice were housed under a controlled temperature of $25 \pm 2^{\circ} \mathrm{C}$, a humidity of $55 \pm 5 \%$, a $12-\mathrm{h}$ light/dark cycle, and free access to water and food. Animals were allowed to adapt to the environment for one week before the experiment. Animals were maintained in accordance with the Guidelines for the Care and Use of Laboratory Animals (29). The present study was approved (approval no. 2018-KY-78) by the Medical Ethics Committee of the First Affiliated Hospital of Zhengzhou University (Zhengzhou, China).

A $70 \%$ hepatic ischemia model was established as previously described (30). Briefly, mice were anesthetized by intraperitoneal injection of pentobarbital sodium $(60 \mathrm{mg} / \mathrm{kg})$. Then, the abdominal cavity was incised along the mid-abdominal line, and microvascular clamps were used to clip the portal vein, hepatic artery, and bile duct resulting in $70 \%$ liver ischemia. Immediately after occlusion, the color of the liver lobes changed from dark red to light red, confirming successful ischemia. After $1 \mathrm{~h}$ of ischemia, the clamp was removed and reperfusion was performed at different time-points. The sham group underwent the same procedure without the blood vessel clamps. The mice were anesthetized by intraperitoneal injection of an overdose of sodium pentobarbital $(100 \mathrm{mg} / \mathrm{kg})$. Blood and liver tissue samples were collected for further analysis after 3, 6, 12, and $24 \mathrm{~h}$ of reperfusion. To determine the effect of miR-140-5p on liver I/R injury, $10 \mathrm{nmol} / \mathrm{g}$ of $\mathrm{miR}-140-5 \mathrm{p}$ agonist and miR-140-5p negative control (NC) (Guangzhou RiboBio Co., Ltd.) were injected into mice via the tail vein $48 \mathrm{~h}$ before I/R.

Cell culture and cell transfection. The mouse hepatic cell line AML12 was purchased from the Cell Bank of the Chinese Academy of Sciences (Shanghai, China), and cultured in Dulbecco's Modified Eagle's Medium/Nutrient Mixture F-12 Medium (DMEM/F12; cat. no. PM150312; Procell Life Science $\&$ Technology Co., Ltd.) supplemented with $10 \%$ fetal bovine serum (FBS; cat. no. 10270-106; Gibco; Thermo Fisher Scientific, Inc.), 1X ITS (cat. no. ITSS-10201; Cyagen Biotechnology, Inc.), 40 ng/ml dexamethasone (cat. no. D8040; Beijing Solarbio Science \& Technology, Inc.), and $1 \%$ penicillin and streptomycin (cat. no. P1400; Beijing Solarbio Science \& Technology, Inc.), in an incubator containing $5 \% \mathrm{CO}_{2}$ at $37^{\circ} \mathrm{C}$. AML12 cells were transfected with $50 \mathrm{nM}$ miR-140-5p mimics (sense, 5'-CAG UGGUUUUACCCUAUGGUAG-3' and antisense, 5'-CUACCA UAGGGUAAAACCACUG-3'); 50 nM miR-140-5p mimics NC (sense, 5'-UCACAACCUCCUAGAAAGAGUA-3' and antisense, 5'-UCUACUCUUUCUAGGAGGUUGU-3'); $50 \mathrm{nM}$ miR-140-5p inhibitor (5'-CUACCAUAGGGUAAAACCACU G-3'), and $50 \mathrm{nM}$ miR-140-5p inhibitor NC (5'-UCUACUCUU UCUAGGAGGUUGU-3') (Hanbio Biotechnology Co., Ltd.) for $48 \mathrm{~h}$ at $37^{\circ} \mathrm{C}$ using Lipofectamine 3000 (cat. no. L300-015; Invitrogen; Thermo Fisher Scientific, Inc.) according to the manufacturer's instructions. The CAPN1 overexpression pcDNA3.1 plasmid and pcDNA3.1 vector (Hanbio Biotechnology Co., Ltd.) were transfected alone or co-transfected with miR-140-5p mimics using Lipofectamine 3000 according to the manufacturer's instructions. Cells were then subjected to hypoxia/reoxygenation (H/R) 48 h post-transfection.

Cell H/R model. AML12 cells were first cultured under normal oxygen concentrations and in a 10\% FBS DMEM/F12 medium. To establish the H/R model, cells were washed twice with PBS (cat. no. P1020; Beijing Solarbio Science \& Technology, Inc.), placed in DMEM/F12 medium without glucose and serum, and were incubated in a tri-gas incubator (Eppendorf $\mathrm{GmbH}$ ) with $5 \% \mathrm{CO}_{2}, 94 \% \mathrm{~N}_{2}$ and $1 \% \mathrm{O}_{2}$ at $37^{\circ} \mathrm{C}$. After $12 \mathrm{~h}$ of hypoxia, the cells were cultured under normal conditions for 3,6 and $12 \mathrm{~h}$ for reoxygenation.

Cell counting Kit-8 (CCK-8) assay. Cell viability was assessed using the CCK-8 assay kit [cat. no. HB-CCK8-2; Hanbio Biotechnology (Shanghai) Co., Ltd.]. Briefly, 5,000 cells from miR-140-5p mimics, mimics NC, miR-140-5p inhibitor and inhibitor NC groups were seeded into individual wells of 96-well plates, and H/R was performed. After reoxygenation, $10 \mu \mathrm{l}$ CCK-8 reagent was added to the AML12 cell medium, and cells were incubated at $37^{\circ} \mathrm{C}$ for $1 \mathrm{~h}$. Thereafter, the absorbance was measured at $450 \mathrm{~nm}$ using a microplate reader (Thermo Fisher Scientific, Inc.).

Lactate dehydrogenase $(\mathrm{LDH})$ release assay. An $\mathrm{LDH}$ release assay was used to assess H/R-induced cell injury as previously described (31). Cellular supernatants from the H/R, transfection, and control groups were collected for the LDH assay (cat. no. A020-2-2; Nanjing Jiancheng Bioengineering Institute), which was performed according to the manufacturer's instructions. Optical density (OD) values were measured at $450 \mathrm{~nm}$ using a microplate reader (Thermo Fisher Scientific, Inc.).

Flow cytometric analysis. Cell apoptosis was determined using an Annexin V-FITC/PI apoptosis detection kit (cat. no. F6012; US Everbright ${ }^{\circledR}$, Inc.) according to the manufacturer's instructions. Briefly, AML12 cells were harvested and resuspended with $100 \mu \mathrm{l}$ binding buffer after being washed twice with cold PBS. Cells were then incubated with $5 \mu \mathrm{l}$ Annexin V-FITC and $5 \mu \mathrm{l}$ PI for $15 \mathrm{~min}$ in the dark at room temperature. Thereafter, $400 \mu$ l binding buffer was added post-incubation. Apoptosis cells was analyzed using a flow cytometric system (BD FACSCanto II, BD Biosciences). 
BD FACSDiva Software v.3.0.1 (BD Biosciences) was used to analyze the results.

Prediction of miR-140-5p targets. StarBase version 2.0 (http://starbase.sysu.edu.cn/index.php), miRDB (http://mirdb. org/index.html), and TargetScan version 7.2 (http://www. targetscan.org) were used to predict potential targets and binding sites of miR-140-5p. Genes predicted by the three databases were considered as potential targets.

Dual-luciferase reporter assay. A dual-luciferase reporter assay was performed to confirm the interaction between miR-140-5p and CAPN1 3'-UTR. Luciferase reporter plasmids (pGL3 backbone) containing wild-type (WT)-CAPN1 or mutant (MUT)-CAPN1 were constructed by Hanbio Biotechnology Co., Ltd. AML-12 cells were co-transfected with miR-140-5p mimics or inhibitors with WT-CAPN1 or MUT-CAPN1 using Lipofectamine 3000. After transfection for $48 \mathrm{~h}$, luciferase activity was detected using a dual-luciferase reporter assay kit (cat. no. F6075; US Everbright ${ }^{\circledR}$. Inc.) according to the manufacturer's instructions. Relative luciferase activity was normalized to Renilla luciferase activity.

Alanine aminotransferase (ALT) and aspartate aminotransferase (AST) assays. ALT and AST levels were assessed in mouse serum using commercial kits (cat. no. C009-2-1 and C010-2-1; Nanjing Jiancheng Bioengineering Institute) according to the manufacturer's instructions.

Hematoxylin and eosin $(H \& E)$ staining. Liver tissues were fixed in $10 \%$ formaldehyde solution at room temperature for $24 \mathrm{~h}$ and embedded in paraffin. The tissue was cut into $5-\mu \mathrm{m}$ sections and stained with an H\&E staining kit (cat. no. G1120; Beijing Solarbio Science \& Technology, Inc.) at room temperature for $5 \mathrm{~min}$ according to the manufacturer's instructions. Images were captured using a light microscope (Olympus Corporation).

Terminal deoxynucleotidyl transferase dUTP nick end labeling (TUNEL) assay. TUNEL staining was performed using One Step TUNEL Apoptosis Assay kit (cat. no. C1086; Beyotime Institute of Biotechnology) according to the manufacturer's instructions. The paraffin-embedded tissue was cut into $5-\mu \mathrm{m}$ sections and stained with TUNEL reaction mixture containing terminal deoxynucleotidyl transferase (TdT), fluorescent labeling solution, and TUNEL detection liquid at room temperature for $1 \mathrm{~h}$ in the dark. DAPI $(10 \mu \mathrm{g} / \mathrm{ml})$ was used to stain the nucleus at room temperature for $10 \mathrm{~min}$ followed by the addition of an anti-fluorescence quenching solution. Images were captured using a fluorescence microscope (Olympus Corporation). For each sample, $>5$ areas were randomly selected for analysis. TUNEL-positive cells in the tissues were quantified using Image-Pro Plus (version 6.0, Media Cybernetics, Inc.).

Western blot analysis. Liver tissues or cells were lysed in radioimmunoprecipitation assay lysis buffer (cat. no. R0010; Beijing Solarbio Science \& Technology, Inc.) according to the manufacturer's instructions. The protein concentration was estimated using a bicinchoninic acid (BCA) protein assay
Table I. Primer sequences for reverse transcription-quantitative PCR.

Gene

Primer sequence (5'-3')

U6

F: CTCGCTTCGGCAGCACA

miR-140-5p

R: AACGCTTCACGAATTTGCGT

IL-6

F: CGCGCAGTGGTTTTACCCTA

R: AGTGCAGGGTCCGAGGTATT

F: AGAGACTTCCATCCAGTTGCC

$\mathrm{IL}-1 \beta$

R: TCCTCTGTGAAGTCTCCTCTCC

TNF- $\alpha$ F: GCTTCAGGCAGGCAGTATCA

R: AGTCACAGAGGATGGGCTCT

F: AGCCGATGGGTTGTACCTTG

R: ATAGCAAATCGGCTGACGGT

F, forward; R, reverse; miR, microRNA; IL, interleukin, TNF, tumor necrosis factor.

kit (cat. no. PC0020; Beijing Solarbio Science \& Technology, Inc.). Equal amounts of $30 \mu \mathrm{g}$ protein sample were separated using 10 or $12 \%$ sodium dodecyl sulfate polyacrylamide gel electrophoresis, and the gel was transferred to $0.45-\mu \mathrm{m}$ polyvinylidene difluoride (PVDF) membranes. The membranes were blocked with $5 \%$ skim milk for $1 \mathrm{~h}$ at room temperature and incubated overnight at $4^{\circ} \mathrm{C}$ with primary antibodies against CAPN1 (1:1,000; cat. no. 10538-1-AP; ProteinTech Group, Inc.), Bcl-2 (1:800; cat. no. 12789-1-AP; ProteinTech Group, Inc.), Bax (1:1,000; cat. no. 50599-2-Ig; ProteinTech Group, Inc.), cleaved caspase-3 (1:1,000; cat. no. WL02117; Wanleibio Co.,Ltd.), and GAPDH (1:5,000; cat. no. 60004-1-Ig; ProteinTech Group, Inc.). The membranes were washed with $1 \%$ TBST $(0.05 \%$ Tween-20; cat. no. CR10301; Monad Biotech Co., Ltd.) before and after incubation with horseradish peroxidase-conjugated goat anti-rabbit antibody $(1: 5,000$; SA00001-2; ProteinTech Group, Inc.) for $1 \mathrm{~h}$ at room temperature. Protein expression was detected using a ChemiDoc ${ }^{\mathrm{TM}} \mathrm{MP}$ Imaging System (Bio-Rad Laboratories, Inc.). Image $\mathrm{Lab}^{\mathrm{TM}}$ software (version 5.2.1; Bio-Rad Laboratories, Inc.) was used to quantify protein expression levels. The relative protein expression was normalized to glyceraldehyde 3-phosphate dehydrogenase (GAPDH).

RNA extraction and reverse transcription-quantitative polymerase chain reaction $(R T-q P C R)$. Total RNA was extracted from the liver tissues and AML12 cells using the Triquick reagent (cat. no. R1100; Beijing Solarbio Science \& Technology, Inc.) according to the manufacturer's instructions, and reverse transcribed into cDNA using the HiScript II Q RT SuperMix (cat. no. R223-01; Vazyme Biotech Co., Ltd.) or miRNA First Strand cDNA Synthesis (cat. no. B532453; Sangon Biotech Co., Ltd.). qPCR analysis was performed using the ChamQ Universal SYBR qPCR Master Mix (cat. no. Q711-02; Vazyme Biotech Co., Ltd.) or miRNA Universal SYBR qPCR Master Mix (cat. no. MQ101-02; Vazyme Biotech Co., Ltd.) according to the manufacturer's instructions. The thermocycling conditions were $95^{\circ} \mathrm{C}$ for $30 \mathrm{sec}, 95^{\circ} \mathrm{C}$ for $5 \mathrm{sec}$, and $60^{\circ} \mathrm{C}$ for $35 \mathrm{sec}$ (40 cycles). The expression of mRNA and miRNA was 

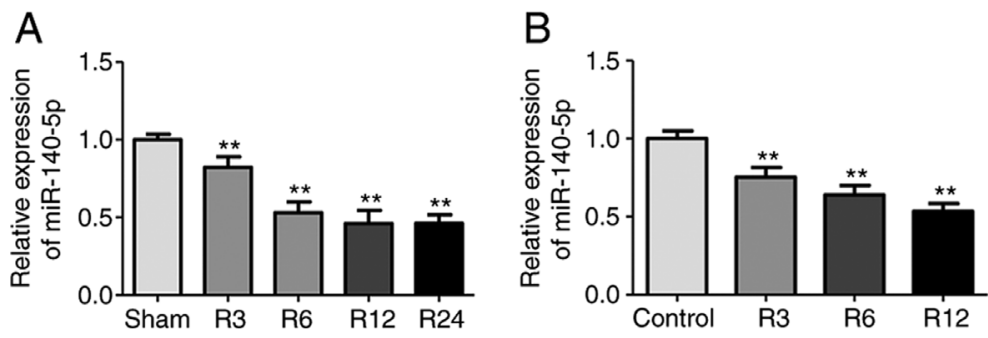

Figure 1. miR-140-5p is decreased in the liver I/R injury mouse model and in AML12 cells subject to H/R. (A) Relative miR-140-5p levels in the liver tissues following I/R and (B) in AML12 cells subjected to H/R at different time-points was detected by reverse transcription-quantitative polymerase chain reaction. ${ }^{* *} \mathrm{P}<0.01$ vs. the sham group and control group. miR, microRNA; I/R, ischemia/reperfusion; H/R, hypoxia reoxygenation; R, reperfusion.

normalized to the expression of GAPDH and U6, respectively. Fold change was calculated by the $2^{-\Delta \Delta \mathrm{Cq}}$ method (32). The sequences of the primers used in this experiment are provided in Table I.

Statistical analysis. SPSS software (version 22.0; IBM Corp.) was used for statistical analyses. All experimental results were independently repeated at least three times. Data are represented as the mean \pm standard deviation (SD). The unpaired Student's t- test was used to analyze the differences between the two groups. One-way analysis of variance (ANOVA) with post-hoc Tukey's multiple comparison test was used to compare differences among multiple groups. $\mathrm{P}<0.05$ was considered to indicate a statistically significant difference.

\section{Results}

miR-140-5p is decreased in the liver I/R injury mouse model and in AML12 cells subjected to $H / R$. To explore the potential role of miR-140-5p in liver I/R, a mouse model of liver I/R injury and AML12 cells subjected to H/R were established. Compared with the sham group, the miR-140-5p expression was significantly downregulated in the liver tissues 6,12 , and $24 \mathrm{~h}$ after reperfusion, but there were no statistical differences among these time-points (Fig. 1A), thus $6 \mathrm{~h}$ was selected as the time-point for further studies. miR-140-5p expression was significantly downregulated in AML12 cells subjected to H/R compared with the control group. Furthermore, miR-140-5p expression decreased gradually as reoxygenation time increased, with the lowest level observed at $12 \mathrm{~h}$ (Fig. 1B). Therefore, $12 \mathrm{~h}$ was selected as the time-point for reoxygenation in the following studies. These results indicated that miR-140-5p may be involved in liver I/R injury.

miR-140-5p regulates cell injury in AML12 cells exposed to $H / R$. To investigate the effect of miR-140-5p on liver I/R injury, an H/R model was established using AML12 cells. Cell viability, LDH release, apoptosis, and expression of inflammatory cytokines were used to evaluate cell injury. miR-140-5p mimic/inhibitor and $\mathrm{NC}$ groups had no effect on cell viability in normal conditions (Fig. S1). miR-140-5p expression was decreased in AML12 cells subjected to H/R but significantly increased after transfection with miR-140-5p mimics and significantly decreased after transfection with miR-140-5p inhibitor (Fig. 2A). Compared with the control group, the viability of AML12 cells significantly decreased after H/R (Fig. 2B), while miR-140-5p overexpression significantly increased the viability of cells exposed to H/R (Fig. 2B). Compared with the control group, LDH release (Fig. 2C) and inflammatory cytokines interleukin (IL)-1 $\beta$, IL-6, and tumor necrosis factor (TNF)- $\alpha$ (Fig. 2D-F) significantly increased after H/R. Overexpression of miR-140-5p significantly decreased LDH release (Fig. 2C) and the expression of inflammatory cytokines (Fig. 2D-F). Flow cytometry and western blotting were performed to evaluate cellular apoptosis. Flow cytometric results indicated that miR-140-5p overexpression significantly decreased cells apoptosis compared with the H/R group (Fig. 2G and H). The levels of apoptosis-related proteins, cleaved caspase- 3 and Bax significantly increased in AML12 cells exposed to H/R, while anti-apoptosis protein Bcl-2 levels significantly decreased compared with those in the control group (Fig. 2I and J). Overexpression of miR-140-5p reversed these changes in apoptosis-related protein expression (Fig. 2I and J). However, compared with the H/R group, inhibition of miR-140-5p expression further reduced cell activity (Fig. 2B) and increased LDH release (Fig. 2C), the expression of inflammatory cytokines (Fig. 2D-F), and cell apoptosis (Fig. 2G-J). These results indicated that miR-140-5p may protect AML12 cells against H/R-induced injury.

miR-140-5p overexpression is decreased liver injury in an $I / R$ injury mouse model. To further elucidate the effect of miR-140-5p on liver I/R injury, mice were pretreated with a miR-140-5p agonist or NC via the tail vein before I/R. RT-qPCR analysis revealed that the miR-140-5p agonist significantly upregulated miR-140-5p expression in the liver tissues compared with that in the I/R group (Fig. 3A). Liver injury was determined by employing serum ALT and AST assays, H\&E staining, TUNEL assays, and apoptosis-related protein and tissue inflammation analysis. Serum ALT and AST levels were significantly decreased in the I/R+ miR-140-5p agonist group compared with those in the I/R group (Fig. 3B and C). H\&E staining revealed that the miR-140-5p agonist reduced the necrotic area compared with the I/R group (Fig. 3D and E). A TUNEL assay revealed a decrease in TUNEL-positive cells (Fig. 3F and G), and western blot analysis revealed decreased levels of pro-apoptosis proteins cleaved-caspase 3 and Bax, and increased anti-apoptosis protein Bcl-2 in the I/R+ miR-140-5p agonist group compared with those in the I/R group (Fig. $3 \mathrm{H}$ and I). miR-140-5p agonist also inhibited inflammatory cytokines IL-1 $\beta$, IL-6, and TNF- $\alpha$ (Fig. 3J-L). These results indicated that miR-140-5p effectively alleviated liver injury caused by I/R. 

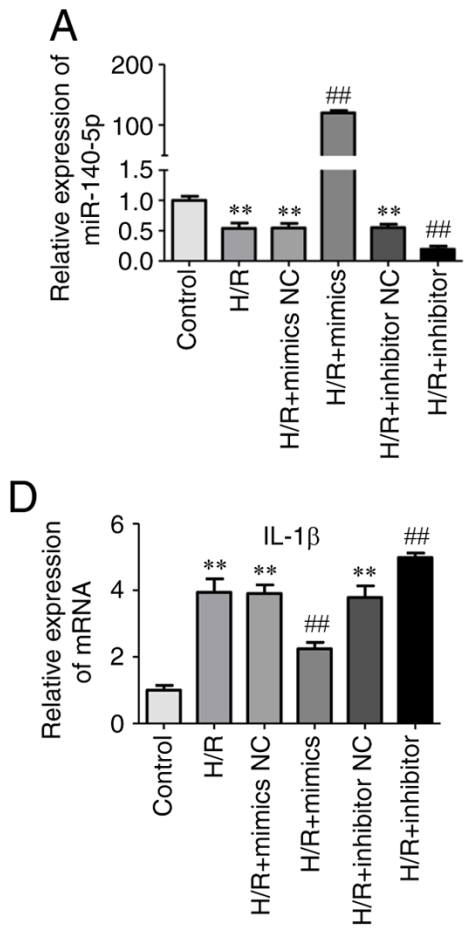

$\mathrm{B}$

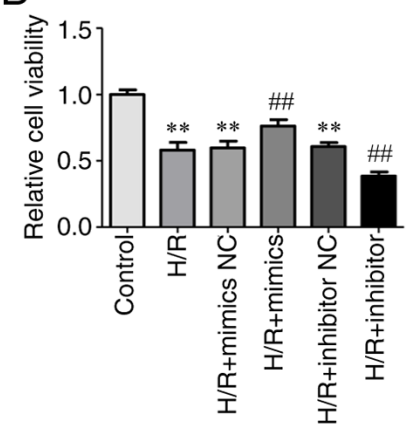

$\mathrm{E}$

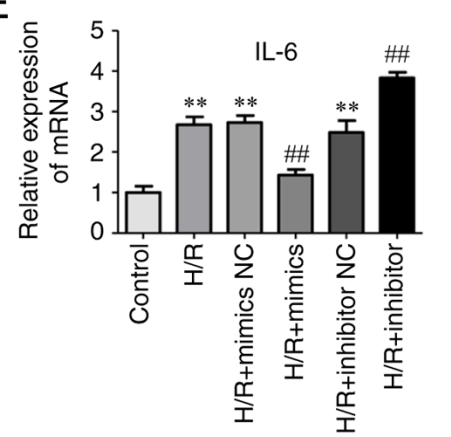

$\mathrm{C}$

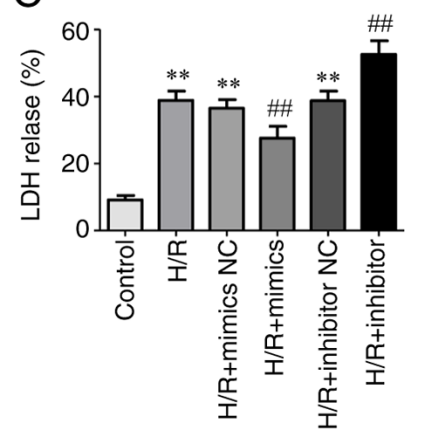

$\mathrm{F}$

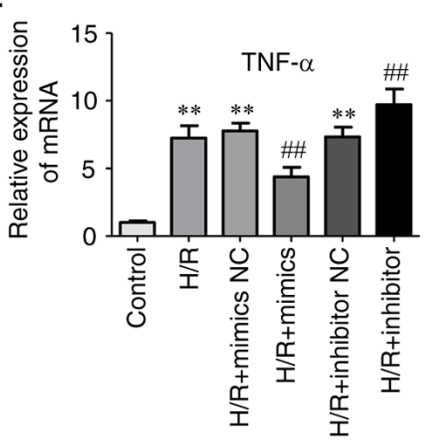

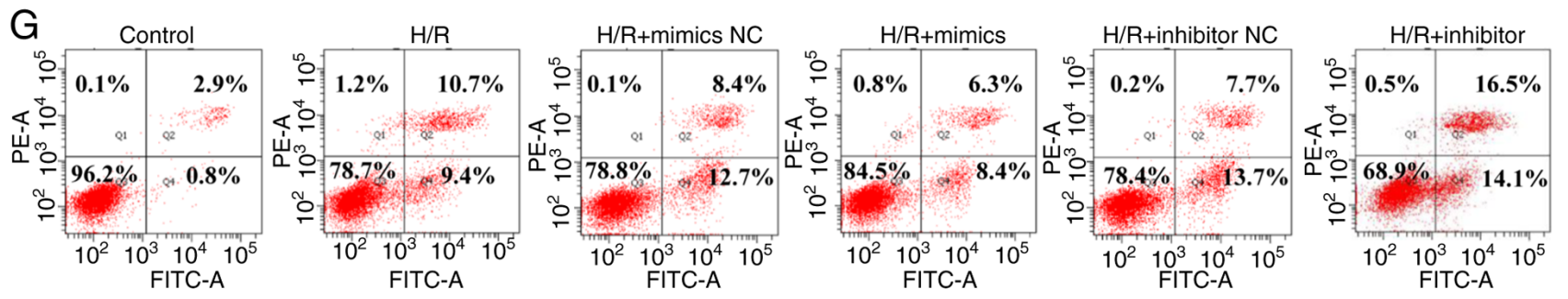
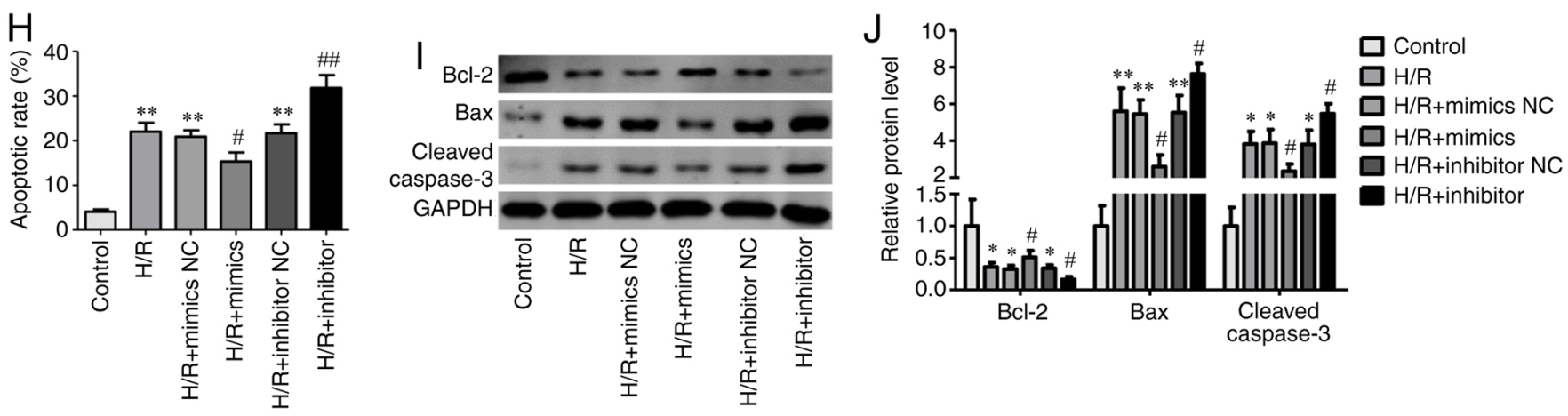

Figure 2. miR-140-5p regulates cell injury in AML12 cells exposed to H/R. (A) Transfection efficiency of miR-140-5p mimics and inhibitor was assessed by RT-qPCR. (B) Effects of miR-140-5p mimics and inhibitors on cell viability after H/R were analyzed by Cell Counting Kit-8 assay. (C) Effects of miR-140-5p mimics and inhibitors on lactate dehydrogenase release after H/R. (D-F) Inflammatory cytokine levels of IL-1 $\beta$, IL-6, and tumor necrosis factor- $\alpha$ were detected by RT-qPCR. (G and H) Cell apoptosis was assessed by flow cytometry. (I) Western blot analysis and (J) quantification of the protein levels of Bax, Bcl-2 and cleaved caspase- 3 . ${ }^{*} \mathrm{P}<0.05$ and ${ }^{* *} \mathrm{P}<0.01$ vs. the control group; ${ }^{*} \mathrm{P}<0.05$ and ${ }^{\# \#} \mathrm{P}<0.01$ vs. the $\mathrm{H} / \mathrm{R}$ group. miR, microRNA; H/R, hypoxia reoxygenation; RT-qPCR, reverse transcription-quantitative polymerase chain reaction; LDH, lactate dehydrogenase; IL, interleukin; TNF, tumor necrosis factor.

miR-140-5p directly targets CAPN1. To explore the mechanism of miR-140-5p in liver $\mathrm{I} / \mathrm{R}$, StarBase, miRDB, and TargetScan were used to predict potential target genes of miR-140-5p. A total of 26 target genes were predicted using the three databases. By detecting the changes in the mRNA expression of target genes after the transfection of miR-140-5p mimics and mimics NC in AML12 cells, 8 of the 26 genes were differentially expressed and, combined with literature reports $(15,33-37)$, CAPN1 was identified as the target gene of miR-140-5p for validation and subsequent verification was performed (Fig. S2). First, the expression of CAPN1 was detected in the mouse liver tissues after I/R injury and in AML12 cells subjected to H/R, and CAPN1 was significantly increased in the $I / R$ and $H / R$ groups (Fig. 4A and B). Then, the interaction between miR-140-5p and CAPN1 was verified; AML12 cells were co-transfected with miR-140-5p mimics or inhibitors with WT-CAPN1 or MUT-CAPN1 luciferase reporter vectors (Fig. 4C). The luciferase reporter assay revealed that overexpression of miR-140-5p attenuated the relative luciferase activity of 

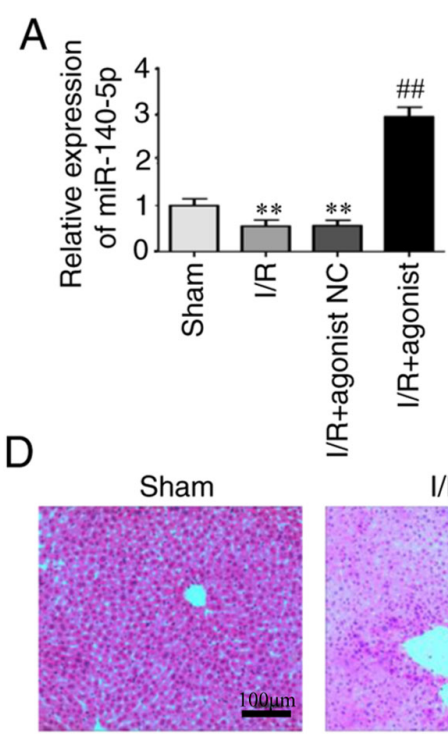

$\mathrm{F}$

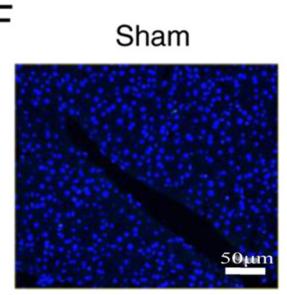

B

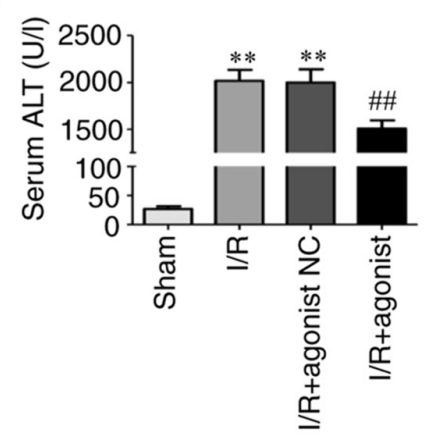

I/R

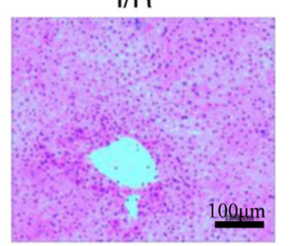

looum
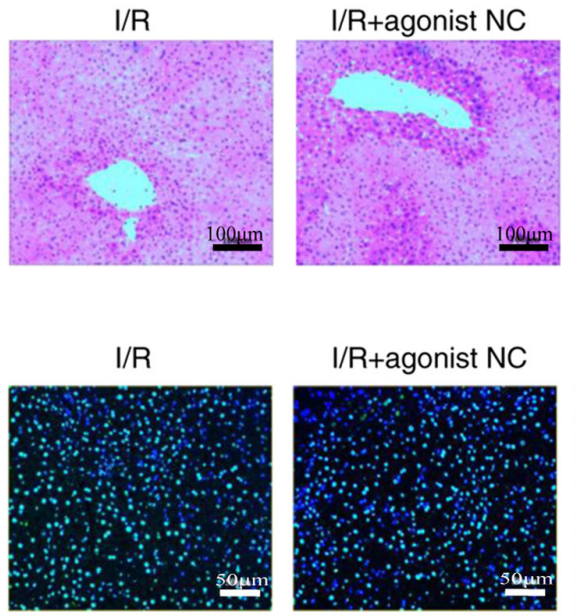

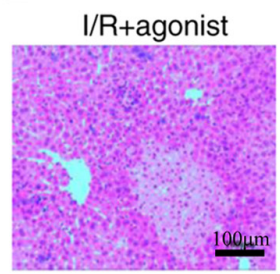

$\mathrm{E}$
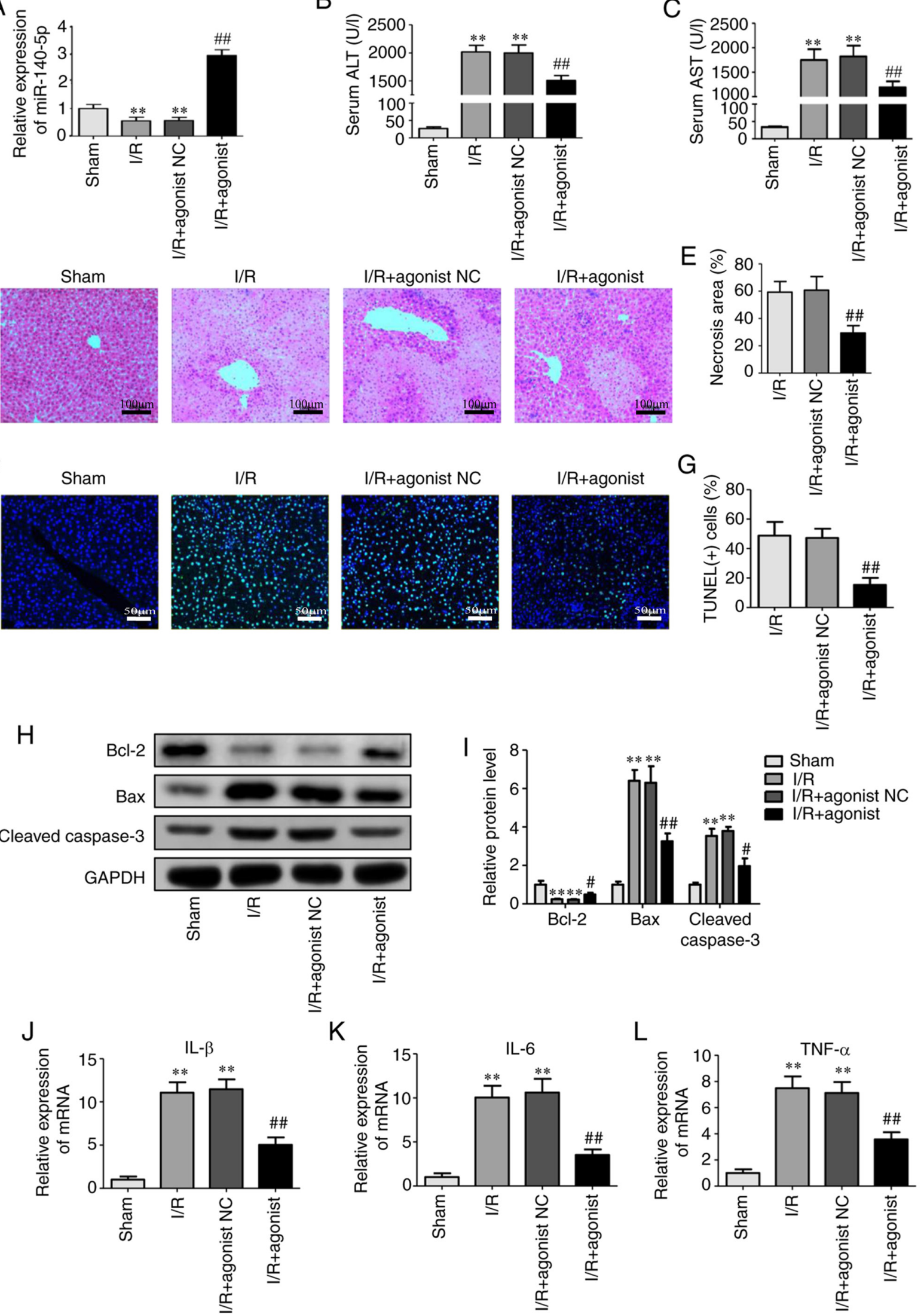

Figure 3. miR-140-5p overexpression decreases liver injury in an I/R injury mouse model. (A) Relative miR-140-5p levels in the liver tissues after injection of miR-140-5p agonist. (B) Serum levels of alanine aminotransferase. (C) Serum levels of aspartate aminotransferase. (D) Hematoxylin and eosin staining of liver sections (Scale bar=100 $\mu \mathrm{m}$ ) and (E) necrotic area analysis. (F) TUNEL staining of hepatocyte apoptosis and (G) quantification of TUNEL(+) cells. Scale bar=50 $\mu \mathrm{m}(\mathrm{H})$ Western blot analysis and (I) quantification of Bax, Bcl-2 and cleaved caspase-3. (J-L) Inflammatory cytokines IL-1 $\beta$, IL-6, and tumor necrosis factor- $\alpha$ was detected by reverse transcription-quantitative polymerase chain reaction. ${ }^{* *} \mathrm{P}<0.01$ vs. the sham group; ${ }^{*} \mathrm{P}<0.05$ and ${ }^{\# \#} \mathrm{P}<0.01$ vs. the $\mathrm{I} / \mathrm{R}$ group. miR, microRNA; I/R, ischemia/reperfusion; ALT, alanine aminotransferase; AST, aspartate aminotransferase; TUNEL, terminal deoxynucleotidyl transferase dUTP nick end labeling; IL, interleukin; TNF, tumor necrosis factor. 

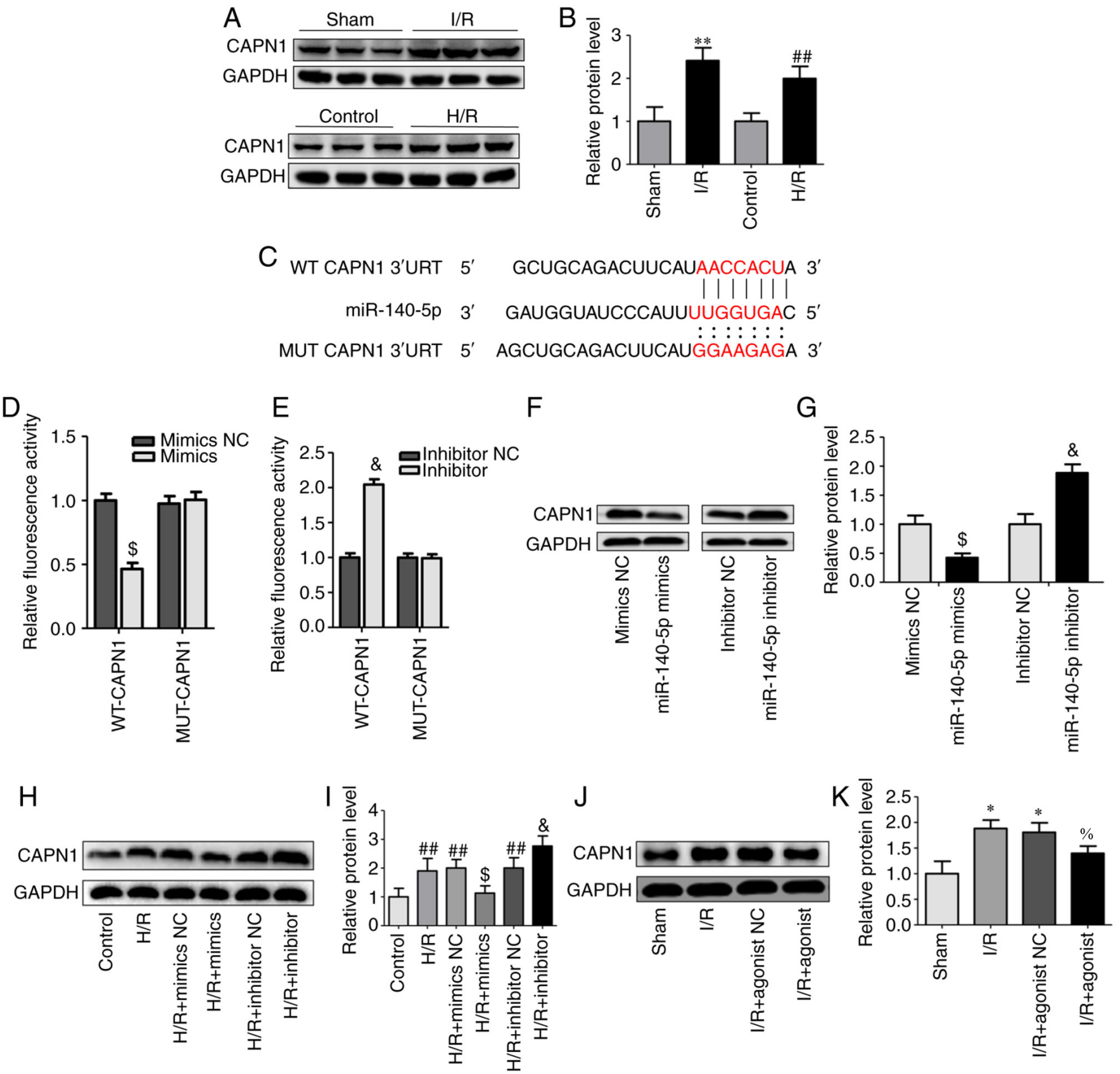

Figure 4. miR-140b-5p directly targets CAPN1. (A) Western blot analysis and (B) quantification of CAPN1 in liver tissues following I/R and AML12 cells following H/R. (C) The binding sites between miR-140-5p and 3'UTR of CAPN1. (D and E) Relative luciferase activity in AML12 cells co-transfected with (D) miR-140-5p mimics or (E) inhibitors with WT-CAPN1 or MUT-CAPN1 luciferase reporter vector. (F) Western blot analysis and (G) quantification of CAPN1 in AML12 cells transfected with miR-140-5p mimics and inhibitors. (H) Western blot analysis and (I) quantification of CAPN1 in AML12 cells transfected miR-140-5p mimics/inhibitor and NCs after H/R. (J) Western blot analysis and (K) quantification of CAPN1 in liver tissues after the injection of miR-140-5p agonist. ${ }^{*} \mathrm{P}<0.05$ and ${ }^{* *} \mathrm{P}<0.01$ vs. the sham group; ${ }^{\%} \mathrm{P}<0.05$ vs. the I/R group; ${ }^{\# \#} \mathrm{P}<0.01$ vs. the control group; ${ }^{\$} \mathrm{P}<0.01$ vs. the mimics $\mathrm{NC}$ group; and ${ }^{\&} \mathrm{P}<0.01$ vs. the inhibitor NC group. miR, microRNA; CAPN1, calpain $1 ; \mathrm{I} / \mathrm{R}$, ischemia/reperfusion; H/R, hypoxia/reoxygenation; UTR, untranslated region; WT, wild-type; MUT, mutant; NC, negative control.

the reporter containing the WT CAPN1-3'-UTR site but not that of the MUT-type CAPN1-3'-UTR (Fig. 4D). However, miR-140-5p inhibition enhanced the luciferase activity of the reporter containing the WT CAPN1-3'-UTR site but not that of the MUT-type CAPN1-3'-UTR (Fig. 4E). To further determine the effect of miR-140-5p on CAPN1, miR-140-5p mimics, inhibitors, and NCs were transfected into AML12 cells, and CAPN1 expression was analyzed. Western blot analyses demonstrated that CAPN1 expression was significantly decreased in the miR-140-5p mimic group
(Fig. 4F and G) and increased in the miR-140-5p inhibitor group compared with that in the respective NC group (Fig. 4F and G). Similar results were observed in AML12 cells exposed to $\mathrm{H} / \mathrm{R}$ and in mice; miR-140-5p mimics inhibited the expression of CAPN1 induced by H/R whereas miR-140-5p inhibitor further increased the expression of CAPN1 induced by H/R (Fig. 4H and I), and miR-140-5p agonist decreased I/R-induced CAPN1 protein expression (Fig. 4J and $\mathrm{K}$ ). These results indicated a targeted relationship between miR-140-5p and CAPN1. 

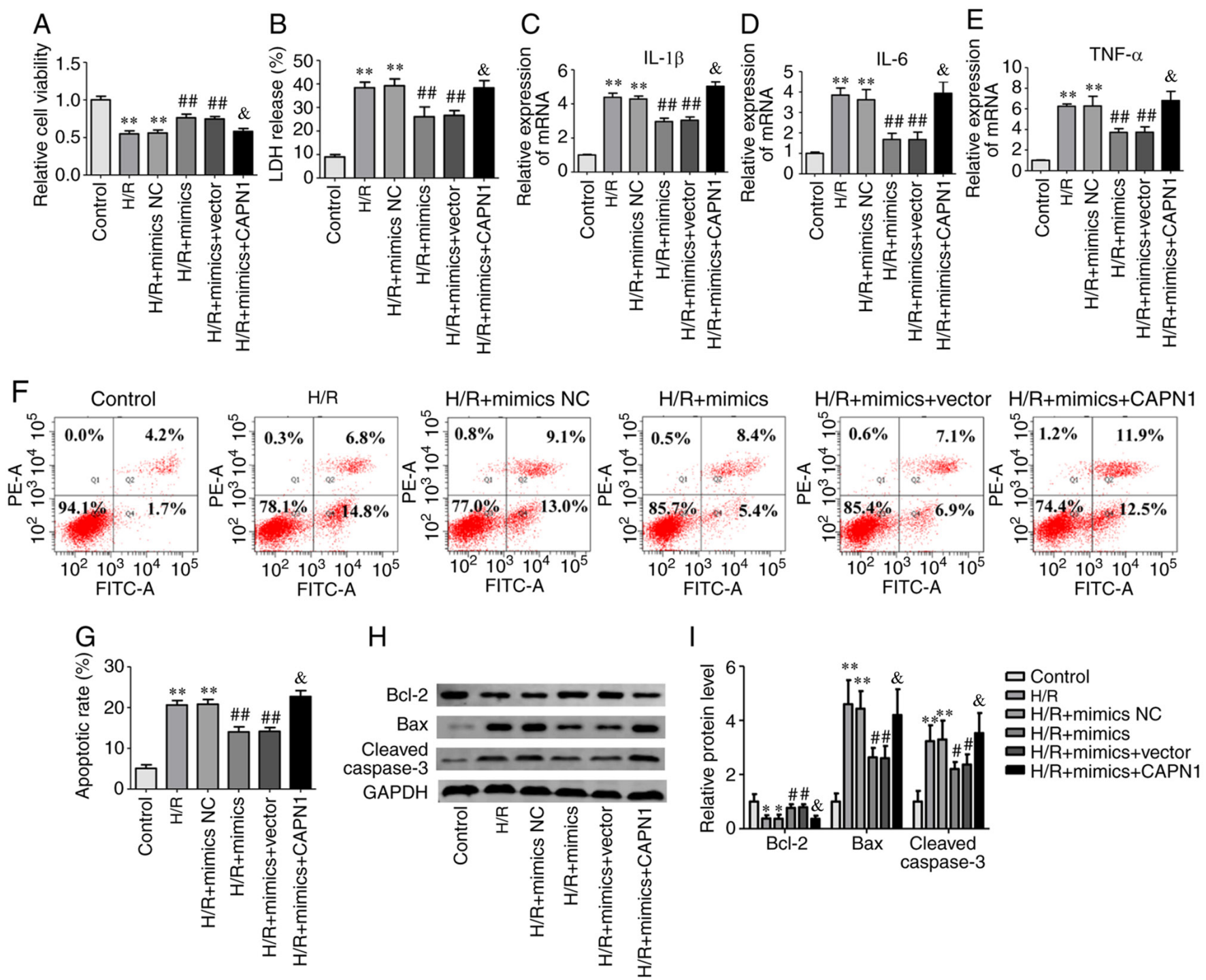

Figure 5. CAPN1 overexpression abrogates the effect of miR-140-5p on H/R-induced cell injury. The AML-12 cells were transfected with miR-140-5p mimics and/or CAPN1 overexpression plasmid in the H/R-induced cell injury model. (A) Relative cell viability, (B) LDH release, (C-E) inflammatory cytokines IL-1 $\beta$, IL-6, and TNF- $\alpha,(\mathrm{F})$ cell apoptosis and (G) apoptotic rate analysis, (H) western blot analysis and (I) quantification of the protein levels of Bax, Bcl-2 and cleaved caspase-3. ${ }^{*} \mathrm{P}<0.05$ and ${ }^{* *} \mathrm{P}<0.01$ vs. the control group; ${ }^{\#} \mathrm{P}<0.05$ and ${ }^{\# \#} \mathrm{P}<0.01$ vs. the $\mathrm{H} / \mathrm{R}$ group; ${ }^{\&} \mathrm{P}<0.05$ vs. the H/R + mimics group. CAPN1, calpain 1; miR, microRNA; H/R, hypoxia/reoxygenation; NC, negative control; LDH, lactate dehydrogenase; IL, interleukin; TNF, tumor necrosis factor.

CAPN1 overexpression abrogates the effect of miR-140-5p on $H / R$-induced cell injury. To determine the effect of CAPN1 on miR-140-5p during H/R-induced cell injury, miR-140-5p mimics were transfected alone or co-transfected with CAPN1 overexpression plasmid into AML12 cells before H/R. Results revealed that the expression of CAPN1 was significantly higher in AML12 cells than that of the vector group after transfection of CAPN1-overexpressing plasmid (Fig. S3), and compared with the miR-140-5p mimic group, overexpression of CAPN1 abrogated the effects of miR-140-5p on $\mathrm{H} / \mathrm{R}$-induced cell injury, which manifested as decreased cell viability (Fig. 5A) and Bcl-2 protein expression (Fig. 5H and I), while LDH release (Fig. 5B), inflammatory cytokine expression (Fig. 5C-E), the apoptotic rate (Fig. 5F and G), and pro-apoptosis proteins cleaved caspase- 3 and Bax expression (Fig. 5H and I) were increased. These results indicated that miR-140-5p alleviated cell injury by inhibiting CAPN1 in AML12 cells.

\section{Discussion}

Liver $\mathrm{I} / \mathrm{R}$ injury is an important pathogenesis of liver transplantation, resection and hemorrhagic shock in clinical practice, and is characterized by oxidative stress, inflammation and apoptosis $(2,3)$. Liver I/R injury can be divided into two distinct pathophysiological processes: Ischemia and reperfusion (3). Currently, effective treatment strategies for hepatic I/R injury are limited due to the lack of basic research that can be translated into clinical applications. Therefore, further studies are needed to identify new targets for alleviating hepatic I/R injury.

Emerging studies have revealed that miRNAs are involved in the process of liver I/R injury. For example, Li et al established that miR-142-3p was downregulated in the liver tissues after I/R, while upregulated miR-142-3p ameliorated liver injury by targeting myristoylated alanine-rich C-kinase substrate (MARCKS) (7). Other miRNAs, such as miR-125b, 
miR-128-3b and miR-450b-5p $(5,6,8)$, are reportedly involved in liver I/R. miR-140-5p has been investigated extensively in tumor research, including glioma, breast and lung cancer, and hepatocellular carcinoma $(13,14,17,18)$. miR-140-5p has also been extensively studied in non-tumor diseases. Han et al revealed that miR-140-5p inhibited apoptosis in brain I/R injury by negatively regulating the expression of the Wnt/b-catenin signaling pathway (38). Wang et al revealed that miR-140-5p attenuated the inflammatory response to brain injury after intracerebral hemorrhage by targeting toll-like receptor 4 (TLR4) (20). Apoptosis and inflammation play important roles in $\mathrm{I} / \mathrm{R}$ injury and are often used as indexes of damage evaluation $(2,30)$. Therefore, it was hypothesized that miR-140-5p may be involved in liver I/R. In the present study, miR-140-5p was significantly downregulated in both mouse liver tissues after I/R and AML12 cells exposed to H/R. In H/R-induced AML12 cell injury, miR-140-5p overexpression markedly increased cell viability, while LDH, apoptosis, and inflammatory factors were decreased. However, cell injury was aggravated after the use of miR-140-5p inhibitors. In the mouse liver I/R injury model, miR-140-5p decreased the levels of ALT and AST in the serum, the necrotic area of the liver, the expression of inflammatory cytokines in liver tissue, and TUNEL-positive hepatic cells, and reversed the changes in apoptosis-related proteins caused by $\mathrm{I} / \mathrm{R}$. The results of in vitro and in vivo experiments demonstrated the protective effect of $\mathrm{miR}-140-5 \mathrm{p}$ in liver I/R.

CAPN1 belongs to the calpains family, and along with other members, is responsible for limited proteolysis of a variety of target substrates (39). Under physiological conditions, calpains are involved in numerous processes such as cytoskeleton remodeling, signal transduction pathway regulation, platelet activation, cell differentiation, and cell apoptosis (39-41). CAPN1 overactivation results in unregulated proteolysis and aberrant activation of signaling cascades, leading to cellular damage and ultimately cell death (42). CAPN1 is activated during heart and brain I/R injury due to increased intracellular calcium concentration, destroying cell structure and increasing apoptosis, reactive oxygen species, and inflammatory cytokines, while inhibiting CAPN1 protects the heart and brain from I/R injury (26-28). In liver I/R injury, CAPN1 activity significantly increased, and CAPN1 inhibition resulted in a significant decrease in liver apoptosis and necrosis (33). In the present study, it was revealed that the expression of CAPN1 was significantly increased in the liver tissues following I/R and in AML12 cells subjected to H/R, and miR-140-5p overexpression inhibited the expression of CAPN1 in vitro and in vivo. The dual-luciferase reporter assay further confirmed that miR-140-5p negatively regulated CAPN1. CAPN1 overexpression inhibited the protective effects of miR-140-5p on AML12 cell injury. The results of the present study indicated that miR-140-5p decreased liver I/R injury by targeting CAPN1.

In conclusion, our present study demonstrated that miR-140-5p alleviated liver I/R injury by negatively regulating the expression of CAPN1 in mouse liver I/R injury and H/R-induced injury in AML12 cells. These findings may contribute to further understanding the mechanism of liver I/R injury and may provide novel insights into liver I/R. miR-140-5p has been extensively studied in tumor and non-tumor diseases and has exhibited favorable results $(13-24,38)$. There is still a large gap in applying miR-140-5p research results to benefit patients clinically. Further research is required to clarify the role of miR-140-5p in liver I/R and other diseases in the clinical setting and to determine how CAPN1 affects liver injury.

\section{Acknowledgements}

Not applicable.

\section{Funding}

The present study was supported by the National Natural Science Foundation of China (grant no. 81971881) and the Medical Science and Technology Project of Henan Provincial Health Commission (grant no. SB201901045).

\section{Availability of data and materials}

All data generated and/or analyzed during the present study are included in this published article.

\section{Authors' contributions}

QY, SC and HT performed the experiments. HY, JZ and XS conducted the literature search and analyzed and interpreted the data. SZ, WG and JL designed the study. QY and SC prepared and wrote the study. All authors have read and approved the final manuscript.

\section{Ethics approval and consent to participate}

The present study was approved by the Medical Ethics Committee of the First Affiliated Hospital of Zhengzhou University.

\section{Patient consent for publication}

Not applicable.

\section{Competing interests}

The authors declare that they have no competing interests.

\section{References}

1. Zhou J, Chen J, Wei Q, Saeb-Parsy K and Xu X: The role of ischemia/reperfusion injury in early hepatic allograft dysfunction. Liver Transpl 26: 1034-1048, 2020.

2. Konishi $\mathrm{T}$ and Lentsch $\mathrm{AB}$ : Hepatic ischemia/reperfusion: Mechanisms of tissue injury, repair, and regeneration. Gene Expr 17: 277-287, 2017.

3. Elias-Miró M, Jiménez-Castro MB, Rodés J and Peralta C: Current knowledge on oxidative stress in hepatic ischemia/reperfusion. Free Radic Res 47: 555-568, 2013.

4. Fabian MR, Sonenberg N and Filipowicz W: Regulation of mRNA translation and stability by microRNAs. Annu Rev Biochem 79: 351-379, 2010.

5. Huang Z, Zheng D, Pu J, Dai J, Zhang Y, Zhang W and Wu Z: MicroRNA-125b protects liver from ischemia/reperfusion injury via inhibiting TRAF6 and NF- $\mathrm{B}$ pathway. Biosci Biotechnol Biochem 83: 829-835, 2019.

6. Mou T, Luo Y, Huang Z, Zheng D, Pu X, Shen A, Pu J, Li T, Dai J, Chen W and Wu Z: Inhibition of microRNA-128-3p alleviates liver ischaemia-reperfusion injury in mice through repressing the Rnd3/NF- $\kappa$ B axis. Innate Immun 26: 528-536, 2020. 
7. Li Y, Gao M,Xu LN, YinLH, Qi Y and Peng JY: MicroRNA-142-3p attenuates hepatic ischemia/reperfusion injury via targeting of myristoylated alanine-rich C-kinase substrate. Pharmacol Res 156: 104783, 2020.

8. Huang Z, Mou T, Luo Y, Pu X, Pu J, Wan L, Gong J, Yang H, Liu Y, Li Z, et al: Inhibition of miR-450b-5p ameliorates hepatic ischemia/reperfusion injury via targeting CRYAB. Cell Death Dis 11: 455, 2020.

9. Chi X, Jiang Y, Chen Y, Yang F, Cai Q, Pan F, Lv L and Zhang X: Suppression of microRNA-27a protects against liver ischemia/reperfusion injury by targeting PPARgamma and inhibiting endoplasmic reticulum stress. Mol Med Rep 20: 4003-4012, 2019.

10. Wienholds E, Kloosterman WP, Miska E, Alvarez-Saavedra E, Berezikov E, de Bruijn E, Horvitz HR, Kauppinen S and Plasterk RH: MicroRNA expression in zebrafish embryonic development. Science 309: 310-311, 2005

11. Tuddenham L, Wheeler G, Ntounia-Fousara S, Waters J, Hajihosseini MK, Clark I and Dalmay T: The cartilage specific microRNA-140 targets histone deacetylase 4 in mouse cells. Febs Lett 580: 4214-4217, 2006.

12. Miyaki S, Nakasa T, Otsuki S, Grogan SP, Higashiyama R, Inoue A, Kato Y, Sato T, Lotz MK and Asahara H: MicroRNA-140 is expressed in differentiated human articular chondrocytes and modulates interleukin-1 responses. Arthritis Rheum 60: 2723-2730, 2009.

13. Zhu D, Lv W, Zhou X, He Y, Yao H, Yu Y, Zhang G and Zhang Q: Long non-coding RNA TMPO-AS1 promotes tumor progression via sponging miR-140-5p in breast cancer. Exp Ther Med 21: 17, 2021.

14. Zhuo E, Cai C, Liu W, Li K and Zhao W: Downregulated microRNA-140-5p expression regulates apoptosis, migration and invasion of lung cancer cells by targeting zinc finger protein 800 . Oncol Lett 20: 390, 2020.

15. Mei J,Liu G, Wang W, Xiao P, Yang D, Bai H and Li R: OIP5-AS1 modulates epigenetic regulator HDAC7 to enhance non-smal cell lung cancer metastasis via miR-140-5p. Oncol Lett 20: 7 , 2020 .

16. Mao Z, Wang Z, Zhang S, Pu Y, Wang J, Zhang T, Long Y, Liu Y, Ma Y and Zhu J: LRP4 promotes migration and invasion of gastric cancer under the regulation of microRNA-140-5p. Cancer Biomark 29: 245-253, 2020.

17. Cai RD, Zhang CC, Xie LL, Wang PC, Huang CX, Chen JL and Lv HT: SNHG1 promotes malignant progression of glioma by targeting miR-140-5p and regulating PI3K/AKT pathway. Cancer Manag Res 12: 12011-12020, 2020.

18. Fan L, Huang X, Chen J, Zhang K, Gu YH, Sun J and Cui SY: Long noncoding RNA MALAT1 contributes to sorafenib resistance by targeting miR-140-5p/Aurora-A signaling in hepatocellular carcinoma. Mol Cancer Ther 19: 1197-1209, 2020.

19. Yang Y, Liu D, Xi Y, Li J, Liu B and Li J: Upregulation of miRNA-140-5p inhibits inflammatory cytokines in acute lung injury through the MyD88/NF-kappaB signaling pathway by targeting TLR4. Exp Ther Med 16: 3913-3920, 2018.

20. Wang S, Cui Y, Xu J and Gao H: miR-140-5p Attenuates Neuroinflammation and Brain Injury in Rats Following Intracerebral Hemorrhage by Targeting TLR4. Inflammation 42: 1869-1877, 2019

21. Wang Y, Shen S, Li Z, Li W and Weng X: MIR-140-5p affects chondrocyte proliferation, apoptosis, and inflammation by targeting HMGB1 in osteoarthritis. Inflamm Res 69: 63-73, 2020.

22. Liao W, Fu Z, Zou Y, Wen D, Ma H, Zhou F, Chen Y, Zhang M and Zhang W: MicroRNA-140-5p attenuated oxidative stress in Cisplatin induced acute kidney injury by activating Nrf2/ARE pathway through a Keap1-independent mechanism. Exp Cell Res 360: 292-302, 2017

23. Liu H, Mao Z, Zhu J, Shen M and Chen F: miR-140-5p inhibits oxidized low-density lipoprotein-induced oxidative stress and cell apoptosis via targeting toll-like receptor 4 . Gene Ther 2020.

24. Sun J, Tao S, Liu L, Guo D, Xia Z and Huang M: miR-140-5p regulates angiogenesis following ischemic stroke by targeting VEGFA. Mol Med Rep 13: 4499-4505, 2016.
25. Demarchi F and Schneider C: The calpain system as a modulator of stress/damage response. Cell Cycle 6: 136-138, 2007.

26. Lu HT, Feng RQ, Tang JK, Zhou JJ, Gao F and Ren J: CaMKII/calpain interaction mediates ischemia/reperfusion injury in isolated rat hearts. Cell Death Dis 11: 388, 2020.

27. Yue RC, Lu SZ, Luo Y, Wang T, Liang H, Zeng J, Liu J and $\mathrm{Hu} \mathrm{HX}$ : Calpain silencing alleviates myocardial ischemia-reperfusion injury through the NLRP3/ASC/Caspase-1 axis in mice. Life Sci 233: 116631, 2019.

28. Zhao H, Xu M and Chu G: Association between myocardial cell apoptosis and calpain-1/caspase-3 expression in rats with hypoxic-ischemic brain damage. Mol Med Rep 15: 2727-2731, 2017.

29. National Research Council: Guide for the Care and Use of Laboratory Animals. National Academies Press, Washington, DC, 2010.

30. Guo WZ, Fang HB, Cao SL, Chen SY, Li J, Shi JH, Tang HW, Zhang Y, Wen PH, Zhang JK, et al: Six-transmembrane epithelial antigen of the prostate 3 Deficiency in hepatocytes protects the liver against ischemia-reperfusion injury by suppressing transforming growth factor- $\beta$-activated kinase 1 . Hepatology 71 : 1037-1054, 2020.

31. Yi Z, Deng M, Scott MJ, Fu G, Loughran PA, Lei Z, Li S, Sun P, Yang $\mathrm{C}, \mathrm{Li} \mathrm{W}$, et al: Immune-responsive gene 1/itaconate activates nuclear factor Erythroid 2-related factor 2 in hepatocytes to protect against liver ischemia-reperfusion injury. Hepatology 72: 1394-1411, 2020.

32. Livak KJ and Schmittgen TD: Analysis of relative gene expression data using real-time quantitative PCR and the 2(-Delta Delta C(T)) method. Methods 25: 402-408, 2001.

33. Kohli V, Madden JF, Bentley RC and Clavien PA: Calpain mediates ischemic injury of the liver through modulation of apoptosis and necrosis. Gastroenterology 116: 168-178, 1999.

34. Li W and He F: Monocyte to macrophage differentiation-associated (MMD) targeted by miR-140-5p regulates tumor growth in non-small cell lung cancer. Biochem Biophys Res Commun 450: 844-850, 2014

35. Kai Y, Peng W, Ling W, Jiebing $\mathrm{H}$ and Zhuan B: Reciprocal effects between microRNA-140-5p and ADAM10 suppress migration and invasion of human tongue cancer cells. Biochem Biophys Res Commun 448: 308-314, 2014.

36. Rothman AM, Arnold ND, Pickworth JA, Iremonger J, Ciuclan L, Allen RM, Guth-Gundel S, Southwood M, Morrell NW, Thomas M, et al: MicroRNA-140-5p and SMURF1 regulate pulmonary arterial hypertension. J Clin Invest 126: 2495-2508, 2016.

37. Güllü G, Peker I, Haholu A, Eren F, Küçükodaci Z, Güleç B, Baloglu H, Erzik C, Özer A and Akkiprik M: Clinical significance of miR-140-5p and miR-193b expression in patients with breast cancer and relationship to IGFBP5. Genet Mol Biol 38: 21-29, 2015

38. Han XR, Wen X, Wang YJ, Wang S, Shen M, Zhang ZF, Fan SH, Shan Q, Wang L, Li MQ, et al: MicroRNA-140-5p elevates cerebral protection of dexmedetomidine against hypoxic-ischaemic brain damage via the Wnt/beta-catenin signalling pathway. J Cell Mol Med 22: 3167-3182, 2018.

39. Sorimachi H, Hata S and Ono Y: Calpain chronicle-an enzyme family under multidisciplinary characterization. Proc Jpn Acad Ser B Phys Biol Sci 87: 287-327, 2011.

40. Kuchay SM and Chishti AH: Calpain-mediated regulation of platelet signaling pathways. Curr Opin Hematol 14: 249-254, 2007.

41. Kakurina GV, Kolegova ES, Shashova EE, Cheremisina OV, Choynzonov EL and Kondakova IV: Relationship between the mRNA expression levels of calpains $1 / 2$ and proteins involved in cytoskeleton remodeling. Acta Naturae 12: 110-113, 2020.

42. Kling A,JantosK,MackH,HornbergerW,DrescherK,Nimmrich V, Relo A, Wicke K, Hutchins CW, Lao Y, et al: Discovery of novel and highly selective inhibitors of calpain for the treatment of Alzheimer's disease: 2-(3-Phenyl-1H-pyrazol-1-yl)-nicotinamides. J Med Chem 60: 7123-7138, 2017.

This work is licensed under a Creative Commons Attribution-NonCommercial-NoDerivatives 4.0 International (CC BY-NC-ND 4.0) License. 